\title{
Resistance of Cement Paste and Its Relationship to Strength under the Corrosive Action of Ambient Media
}

\author{
Weilun Wang ${ }^{1}$, Shiqun $\mathrm{Li}^{2}$, Jiashan $\mathrm{Hu}^{2}$, Feng Xing ${ }^{1}$ \\ ${ }^{1}$ Guangdong Provincial Key Laboratory of Durability for Civil Engineering, Shenzhen University, Shenzhen, China \\ ${ }^{2}$ School of Materials Science and Engineering, University of Jinan, Jinan, China \\ Email: wang_weilun@hotmail.com
}

Received February 13, 2012; revised April 19, 2012; accepted April 26, 2012

\begin{abstract}
In this study, we measured the resistances (test frequency $837.8 \mathrm{~Hz}$ ) of the paste of Portland cement (PC) and phosphoaluminate cement (PALC) subjected to different types of corrosion and different numbers of freeze-thaw cycles. This study aimed to improve understanding of the changing characteristics of paste resistance from both micro and macro perspectives by associating changes in the paste microstructure with changes in the paste mechanical strength using X-ray diffraction (XRD), nuclear magnetic resonance (NMR), and other methods. Our results showed that changes in the paste resistance under the corrosive action of ambient media could signal the deterioration of paste structure and loss of paste strength. Continuous hydration reactions within the paste were found to render it more dense and increase its resistance. Invasion of corrosive ions was found to continue to increase paste resistance if the structure of the cement paste was not destroyed. Otherwise, paste resistance would decrease. Corrosive media were found to cause the dispersion of hydrated gels with certain degree of polymerization. Because spatial resistance was found to cause difficulty in the transportation of ion clusters, the decreases in resistance caused by long-term corrosion might be reduced due to a compensation effect. This effect was found to be related to the severity of structural damage to the paste. The magnitudes of corrosive effects of chemical media on the radicals in the cement paste structure were found to occur in the following order: $\mathrm{SiO}_{4}>\mathrm{AlO}_{4}>\mathrm{PO}_{4}$. The resistance and strength of the PC was always lower than those of PALC. In addition, losses of resistance and strength by PALC were mainly due to deterioration of the radical structure of $\mathrm{AlO}_{6}$.
\end{abstract}

Keywords: Cement Paste; Ambient Media; Resistance Characterization; Microstructure; Strength

\section{Introduction}

To determine the hydrous state and properties of cement paste by response of electrical features is both effective and fast. For example, the AC impedance of the paste can be used to determine both early-stage and middlestage hydration mechanisms [1-3]. AC impedance spectroscopy allows non-destructive monitoring of the dispersity of fibers in fiber-reinforced electrically conductive cement-based composites [4]. The combined use of timedomain reflectometry (TDR) and AC-impedance spectroscopy can improve the response of fresh fiber reinforced cement-based composites to impedance [5]. The eddy current method is a very good approach to the study of early contractions in concrete [6]. The galvanostatic method can be used to measure the electrical properties and microstructure of cement-based composites [7]. The resistance of hydrated cement paste can reflect the hardening process and texture $[8,9]$. Hardened hydrated cement paste is a relatively condensed perforated system that can be damaged by freeze-thaw cycles, fresh water, seawater, and other corrosive media, all of which can reduce its lifetime. The mechanical strength and microstructure of the paste are closely related to changes in its resistance. In this way, changes in paste resistance in external electric fields can be used to evaluate the endurance of the material [10]. This study focused on investigating the resistance of the new PALC and PC under the effects of chemical corrosion and freeze-thaw cycles [11]. Its aim is to foster understanding of the rules behind changes in paste resistance changes the action of corrosive media at the micro-structural level and to correlate changes in the micro-structure of the paste to changes in its mechanical strength under the influence of corrosive media via resistance properties. This will provide a basis for the development of fast, non-destructive methods for the assessment of changes in paste quality.

\section{Experiments}

\subsection{Materials}

This study used PALC and PC. Artificial seawater was used as chemical corrosive media (see Table 1 for composition) and $\mathrm{MgSO}_{4}$ solution at a mass concentration of 
Table 1. Chemical composition of artificial seawater (g/l).

\begin{tabular}{ccccc}
\hline $\mathrm{NaCl}$ & $\mathrm{MgCl}_{2} \cdot 6 \mathrm{H}_{2} \mathrm{O}$ & $\mathrm{Na}_{2} \mathrm{SO}_{4}$ & $\mathrm{CaCl}_{2}$ & $\mathrm{KCl}$ \\
\hline 24.5 & 11.1 & 4.1 & 1.2 & 0.7 \\
\hline
\end{tabular}

$5 \%$ in water. Chemically pure agents were used in all solution preparations: $\mathrm{NaCl}, \mathrm{Na}_{2} \mathrm{SO}_{4}, \mathrm{CaCO}_{3}, \mathrm{MgNO}_{3}$, $\mathrm{MgCl}_{2}, \mathrm{CaCl}_{2}$, and $\mathrm{MgSO}_{4}$.

\subsection{Sample Preparation and Testing Methods}

PALC was prepared by our laboratory. PC was manufactured by Shandong Cement Factory using a rotary kiln. Fineness was controlled by filtering $4 \mathrm{w} / \%$ sieve residue through $80 \mu \mathrm{m}$ sieve. PC and PALC were molded to $20 \times$ $20 \times 20 \mathrm{~mm}$ net paste samples. After 28 days of standard curing, they were moved to artificial seawater, moved to $5 \% \mathrm{MgSO}_{4}$ solution, or subjected to freeze-thaw cycles. After the samples were soaked for the designated lengths of time or subjected to designated numbers of freezethaw cycles, a HP4191 radiofrequency impedance analyzer (Agilent Technologies, US) was used to measure the resistances of cement net pastes under different conditions. The testing frequency was $837.8 \mathrm{~Hz}$.

\section{Results and Analysis}

\subsection{Resistance of Cement Paste after Freeze-Thaw Cycles}

Figures 1 and 2 illustrate changes in the resistance and the compressive strength of the paste samples over time after freeze-thaw cycles. The changes in the resistance of cement paste are mainly influenced by two factors. First, as the cement continues to hydrate, the paste structure becomes condensed and the amount free water is reduced, leading to increased resistance. Second, after the freezethaw cycles, the capillary water in the cement net paste freezes and expands, producing micro-fractures. These micro-fractures grow as the number of freeze-thaw cycles increases, permitting further invasion of liquid media. This increases the concentration of transportable ion impurities and decreases resistance decrease. At this point, the dispersion of the hydrated gel can usually compensate for the decreased resistance.

The parameters measured at the end of the 28 days of standard curing were used as values at cycle 0 . When the number of freeze-thaw cycles increased to 50, the resistances of both types of cement net paste showed a decreasing trend (Figures 1 and 2). However, the resistances reached peak values at cycle 100, which corresponded to peak values in the compressive strengths. This suggested that during the period from cycle 50 to cycle 100 , the paste structure condensed, and hydration played a dominant role in this process. After the $100^{\text {th }}$ cycle, the resistances showed a decreasing trend, and so did the strengths,

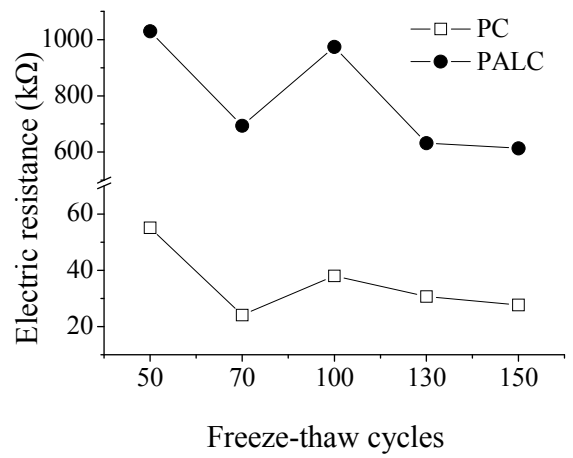

Figure 1. Resistance of cement paste subjected to freezethaw cycles.

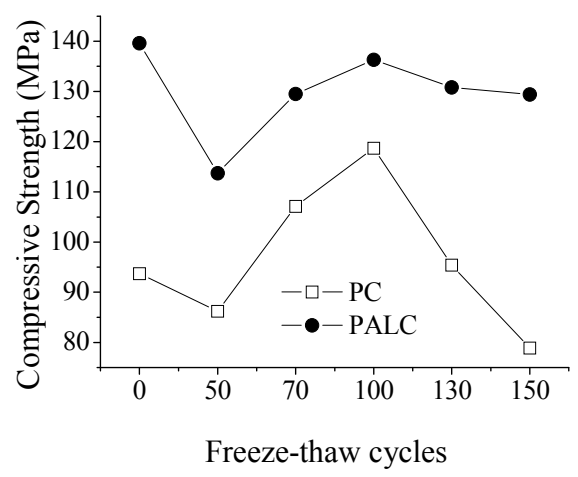

Figure 2. Compressive strength of cement paste subjected to freeze-thaw cycles.

especially that of the PC sample. This suggested substantial increase and expansion of the micro-fracture in the paste due to freeze-thaw. During all freeze-thaw cycles, the resistance of the PALC paste was consistently $15-20$ times higher than that of the PC paste (Figure 1). The same trend was observed for compressive strength (Figure 2). Such results suggest that the structure of the PALC paste became condensed with a relatively small number of fractures in PALC paste, which expanded slowly. These fractures caused the high tolerance of PALC to freeze-thaw cycles. At cycle 150, there was still no substantial decrease in strength.

Resistance of cement paste Figure 3 shows an XRD analysis of the hydrated paste before and after the freezethaw cycles. As illustrated in Figure 3(a), for PC, the diffraction peaks of hydration products calcium hydroxide and ettringite increased after several freeze-thaw cycles, whereas the diffraction peaks of clinker compounds $\mathrm{C}_{2} \mathrm{~S}$ and $\mathrm{C}_{3} \mathrm{~S}$ continued to decrease as the process continued. Figure 3(b) shows that, for PALC, after several freezethaw cycles, except for the clinker compounds of $d$ values $0.374 \mathrm{~nm}$ and $0.296 \mathrm{~nm}$ showed a decrease in the diffracttion peak. Compounds with $\mathrm{d}$ values of $0.280 \mathrm{~nm}$ showed a decrease the diffraction peak but also a broadening of the peak base. The diffraction intensities of hydration products with $\mathrm{d}$ values of $1.419 \mathrm{~nm}$ and $0.718 \mathrm{~nm}$ increased 

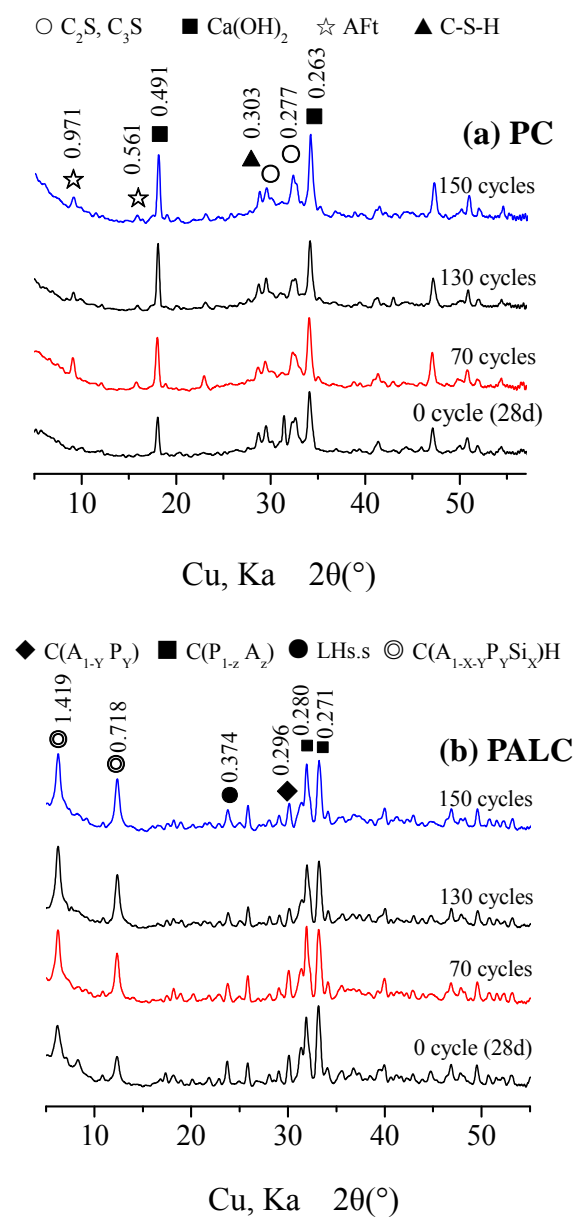

Figure 3. XRD of cement hydrated paste subjected to freezethaw cycles.

after several freeze-thaw cycles. This suggested that, during the freeze-thaw cycles, although the hydration of the cement paste was limited during the freezing period, the clinker compounds continued to be hydrated during the thawing period. This was particularly notable before the $130^{\text {th }}$ cycle. It was the continuing hydration of the cement that led to the increase in the resistance of the cement paste, partly counteracting the decrease in the resistance caused by micro-fractures. This resulted in the fact that the decrease in resistance between freeze-thaw cycle 100 and 150 was not pronounced. However, during the same time, the strength of PC paste dramatically decreased, suggesting substantial dispersion of hydrated gel in the PC paste.

\subsection{Under the Effect of Chemical Corrosion}

Figure 4 shows the resistances of net paste samples during standard curing, after corrosion by seawater and by $\mathrm{MgSO}_{4}$ solution at the testing frequency, $837.8 \mathrm{~Hz}$. The resistance of cement paste under the effects of chemical corrosion is thought to be affected by three main factors.
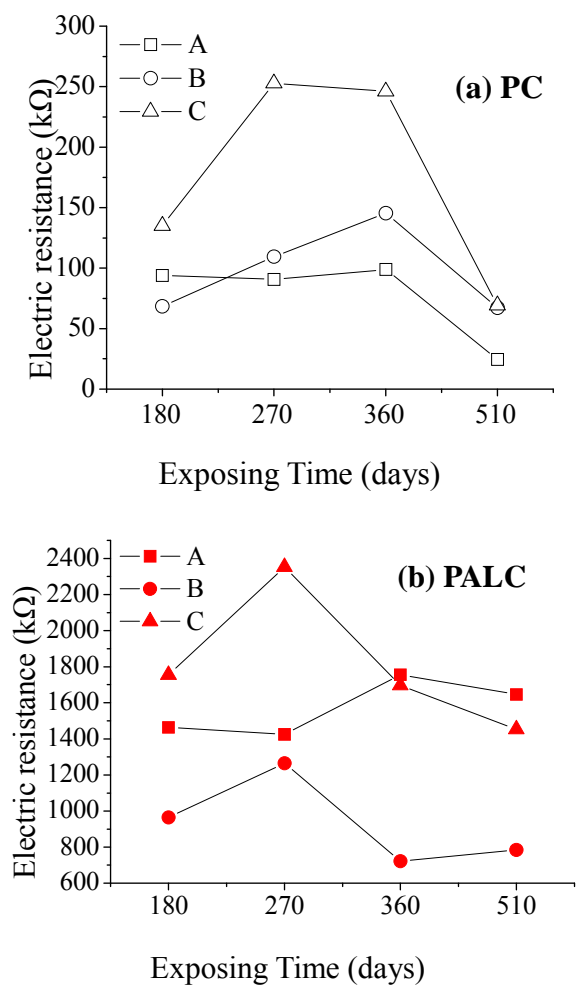

Figure 4. Changes in the paste resistance over time. ((a) Standard curing; (b) Corrosion by seawater; (c) Corrosion by $5 \mathrm{w} / \% \mathrm{MgSO}_{4}$ solution).

First, the continuous hydration reaction in the paste condenses structurally. This leads to increased resistance. Second, the invading external ions react with the hydration products in the paste and generate expansive products. When the amount of the generated expansive products is small, there is not enough internal stress to damage the structure of the cement paste, and the resistance of the paste continues to increase. However, with more severe chemical corrosion, the increasing amounts of internal stress produce micro-fractures in the paste, and corrosive ions continue to be transported into the cement along the micro-fracture. This leads to a decrease in the cement paste. Third, corrosive media disperses the polymerization of the hydrated gel in the paste and causes an increase in the concentration of free radicals in the paste. Due to the difficulty of transporting ions by spatial resistance, the decrease in paste resistance is counteracted to some extent. These three factors interact with each other and have different effects over time (Figure 4). The resistance of $\mathrm{PC}$ paste under the corrosive effects of seawater and $\mathrm{MgSO}_{4}$ solution is always higher than that of the sample under standard curing. This is because the corrosive media cause dispersion of the hydrated gel and so increase the number of anion clusters in the cement paste. This renders the transportation of ions more difficult and causes an increase in the resistance of the paste. On day 
510, the expansion of micro-fractures in the cement was found to play dominant role in the aggravation of corrosion (Figure 5).

Under the action of corrosive media, the resistance of PALC paste was consistently higher than that of the PC paste (Figure 4); it was the same when the cement pastes were subjected to freeze-thaw cycles (Figure 1). Throughout the corrosive process, the resistance of PALC paste was always higher than that of PC paste by about one order of magnitude. This suggests that the texture of PALC paste was condensed and that the condensed hydration system strengthened the tolerance of the paste to corrosive ambient media.

Interestingly, the resistance of the sample exposed to $\mathrm{MgSO}_{4}$ solution was higher than that of the sample exposed to seawater and even higher than that of the sample exposed to standard curing conditions. This could be explained by the changes in the mechanical strength (Figure 5). After 270 days of corrosion by seawater or $\mathrm{MgSO}_{4}$ solution, the mechanical strength of the PC paste dropped substantially due to dispersion of hydrated calcium silicate gel and the expansion of micro-fractures. The resistances of samples exposed to these two conditions were both lower than that of samples exposed to

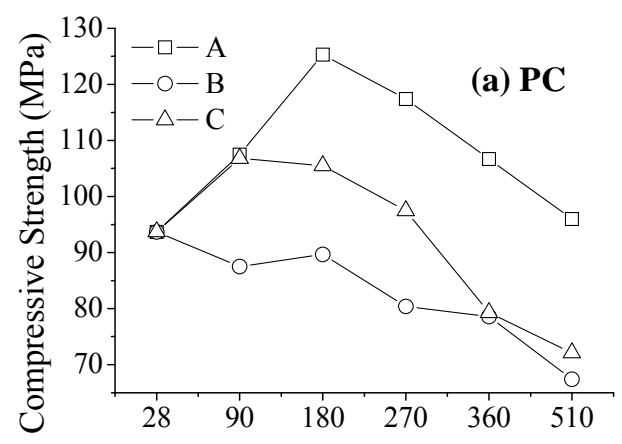

Exposing Time (days)

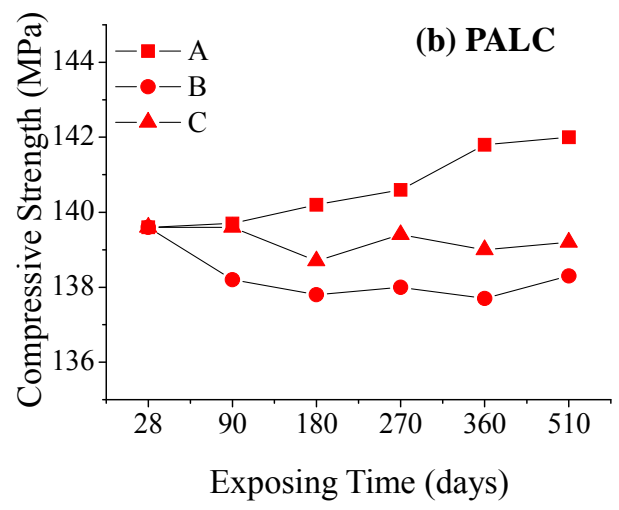

Figure 5. Changes in the compressive strength of cement net paste samples relative to time of corrosions (A, B, and C are the same as in Figure 4). standard curing conditions. Although the mechanical strength of PALC paste did not substantially decrease after 180 days of either type of corrosion, the resistance was still lower than that of samples exposed to standard curing conditions. Combined with the fact that the resistance of PALC paste under the corrosive effect of $\mathrm{MgSO}_{4}$ solution was higher than under the corrosive effect of seawater, it could be deduced that the transportation capability of the negatively charged $\mathrm{SiO}_{4}$ groups was far lower than that of $\mathrm{Cl}^{-}$. Therefore, during the later stages of chemical corrosion, large amounts of dispersion-bound anions and negatively charged $\mathrm{SiO}_{4}$ groups together resulted in higher resistance under the corrosive effect of $\mathrm{MgSO}_{4}$ solution than under either the corrosive effect of seawater or under standard curing conditions.

The marked dispersion of siloxane polymers in the hydrated calcium silicate gel caused by chemical corrosion can be observed from the NMR spectra ( $\left.{ }^{29} \mathrm{Si}-\mathrm{NMR}\right)$ shown in Figure 6. By comparing Figures 6(a) (standard curing), (b) (corrosion by seawater) and (c) (corrosion by $\mathrm{MgSO}_{4}$ solution), it can be seen that, for PC paste under corrosive conditions, the absorption peak of monomer $\mathrm{Q}_{0}$ at $-70.85 \mathrm{ppm}$ and that of dipolymer $\mathrm{Q}_{1}$ at $78.45 \mathrm{ppm}$ exhibited noticeable chemical shifts in the range 0.43 $1.19 \mathrm{ppm}$, and the absorption peak of tripolymer $\mathrm{Q}_{3}$ at $-82.99 \mathrm{ppm}$ and a small number of polymers of higher degree at $-94.5 \mathrm{ppm}$ disappeared. The intensity of the resonance stopband decreased from $4.4 \times 10^{7} \mathrm{CPS}$ to 3.2 $\times 10^{7} \mathrm{CPS}$, by about $27.3 \%$, and the width of the resonance stopband also increased by far more than $0.3 \mathrm{ppm}$. This suggests that the corrosive effect of chemical media not only disrupted the aggregation state of the $\mathrm{SiO}_{4}$ tetrahedron, but also reduced the degree of polymerization of siloxane tetrahedron. The detachment of several powerful anion clusters and the large decrease in the order led to severe deformation of $\mathrm{SiO}_{4}$, which inevitably caused several weak bonds in the structure. These above changes in the paste structure led to a decrease in its strength, but the dispersion did not substantially alleviate the decrease in the resistance of the paste day 360 to day 510. This suggests that the deterioration was the micro-structure of the PC paste was relatively severe.

Figure 7 shows the ${ }^{31} \mathrm{P}$ NMR spectra of PALC paste on day 270. By comparing the ${ }^{31} \mathrm{P}$ NMR spectrum under standard curing conditions (Figure 7(a)), under corrosion by seawater (Figure 7(b)), and under corrosion by $\mathrm{MgSO}_{4}$ solution (Figure 7(c)), it can be seen that, the ${ }^{31} \mathrm{P}$ resonance absorption band at $2.75 \mathrm{ppm}$ exhibited chemical shifts in the range $0.24-0.4 \mathrm{ppm}$ under both types of corrosive conditions. These shifts were mostly toward lower frequencies. The adjacent symmetric sidebands were narrower than under standard curing conditions, by $0.38-0.15 \mathrm{ppm}$ for seawater and by $0.55 \mathrm{ppm}$ for $\mathrm{MgSO}_{4}$. However, under corrosive conditions, the absorption 


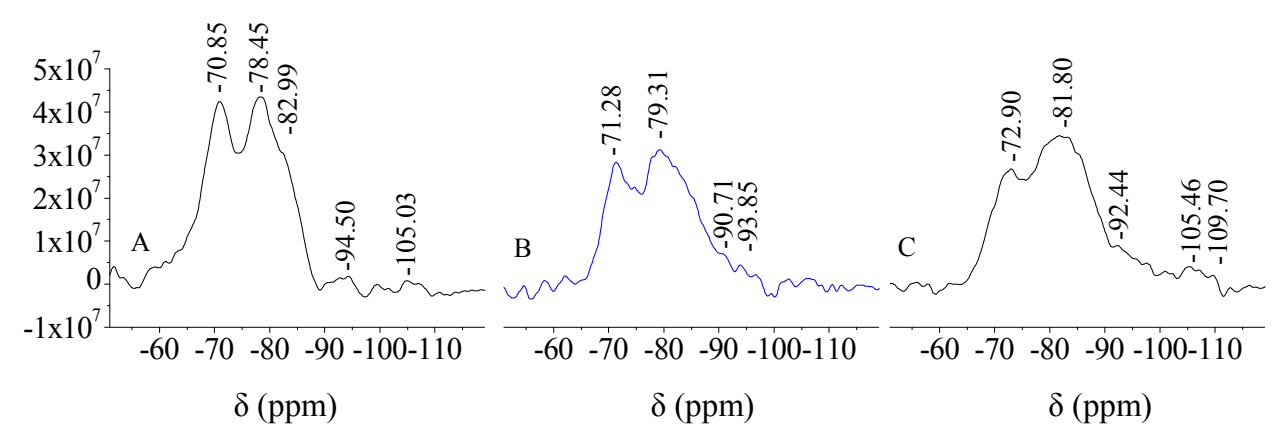

Figure 6. The ${ }^{29} \mathrm{Si}-\mathrm{NMR}$ spectra of PC cement paste on day 270 (A, B, and C are the same as in Figure 4).

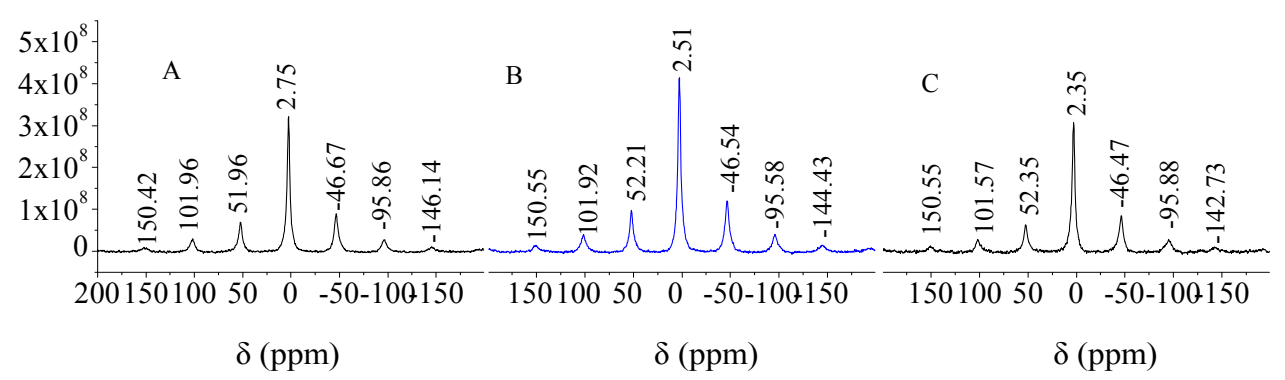

Figure 7. The ${ }^{31} \mathrm{P}$-NMR spectra of PALC cement paste on day 270 (A, B, and C are the same as in Figure 4).
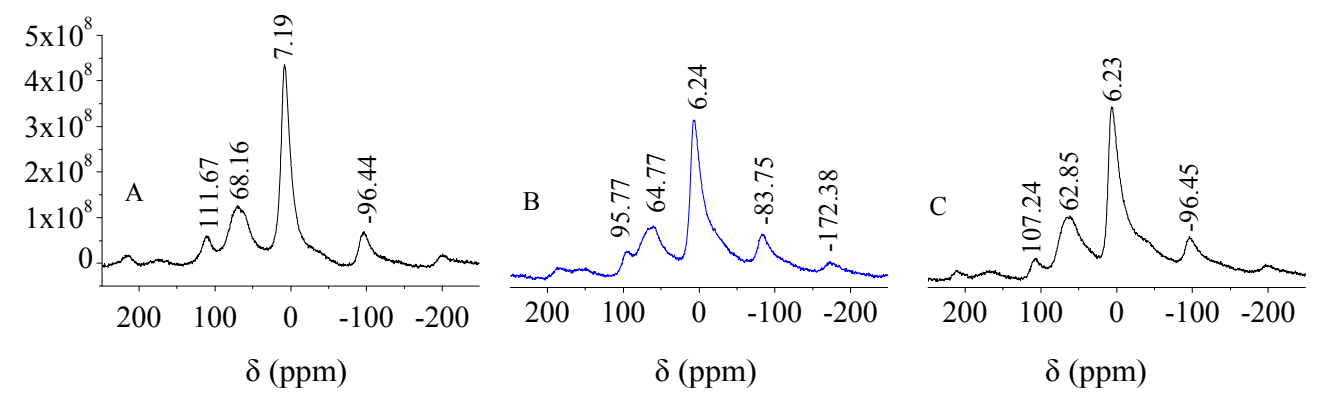

Figure 8. The ${ }^{27} \mathrm{Al}$ NMR spectra of PALC cement paste day 270 (A, B, and C are the same as in Figure 4).

bands remained symmetrical, and there was no notable loss in the intensity or broadening of the spectral bands. However, the absorption band at $2.51 \mathrm{ppm}$ showed a $27.3 \%$ increase (from $3.2 \times 10^{8}$ to $4.2 \times 10^{8} \mathrm{CPS}$ ), and the absorption band at $2.35 \mathrm{ppm}$ in Figure 7(c) was sharper than the absorption band at 2.75 ppm in Figure 7(a) while the count remained the same. This suggests that although corrosion can lead to certain changes in the chemical environment of ${ }^{31} \mathrm{P}$, the corrosive effects of the seawater and the $\mathrm{MgSO}_{4}$ solution on the $\mathrm{PO}_{4}$ group in the PALC cement paste were still not notable during this investigation.

Figure 8 shows the ${ }^{27} \mathrm{Al}$ NMR spectra of PALC paste on day 270. The 6-coordinate $\mathrm{AlO}_{6}$ group shifted from $7.19 \mathrm{ppm}$ under standard curing conditions to $6.24 \mathrm{ppm}$ (seawater) and $6.23 \mathrm{ppm}\left(\mathrm{MgSO}_{4}\right.$ solution). The intensity of the absorption band also decreased by about $27.3 \%$ (from $4.36 \times 10^{8}$ to $3.17 \times 10^{8} \mathrm{CPS}$ ), and the band showed a tendency to deform and broaden. This suggested that, in corrosive media, the structure of some hydration products can be destroyed and that this is the main cause of decreases in strength and resistance. However, from day 360 to day 510 , the drop in resistance was relatively mild. In this way, it could be deduced that the damage to the structure of the PALC paste from corrosion was far less severe than the damage to $\mathrm{PC}$ paste.

\section{Conclusions}

1) Changes in the resistance of the paste in corrosive ambient media can signal the deterioration of paste structure and the loss of mechanical strength.

2) During repeated freeze-thaw cycles, the change in resistance of cement paste is influenced by two factors. Continuous hydration of the cement condenses the paste structure and increases resistance. Aggravation of the invasion of liquid media due to the expansion of microfractures leads to increases in the concentration of transportable ion impurities and decreases resistance. 
3) The resistance of cement paste during chemical corrosion is mainly influenced by three factors. First, the continuous hydration reaction in the paste increases resistance. Second, the invasion of corrosive ions, when not severe enough to damage the structure of the cement paste, leads to a continuous increase in resistance; otherwise, it causes the resistance of the paste to drop. Third, the corrosive media will cause the dispersion of hydrated gel of certain degree of polymerization in the paste, and resistance decrease of the corroded paste over a relatively long period time may be compensated by the effect of spatial resistance.

4) The magnitude of the corrosive effects of chemical media on the radicals in the cement paste structure showed the following order: $\mathrm{SiO}_{4}>\mathrm{AlO}_{4}>\mathrm{PO}_{4}$. The resistance and strength of PC paste was always found to be lower than that of PALC paste.

\section{Acknowledgements}

This work was supported by National Natural Science Foundation of China (51078236) and the National Science Fund of China (50925829) for Distinguished Young Scholars.

\section{REFERENCES}

[1] Y.-M. Kim, J.-H. Lee and S.-H. Hong, "Study of Alinite Cement Hydration by Impedance Spectroscopy," Cement and Concrete Research, Vol. 33, No. 3, 2003, pp. 299-304. doi:10.1016/S0008-8846(02)00944-4

[2] Y. S. Liao, X. S. Wei and G. W. Li, "Early Hydration of Calcium Sulfoaluminate Cement through Electrical Resistivity Measurement and Microstructure Investigations," Construction and Building Materials, Vol. 24, No. 4, 2011, pp. 1572-1579. doi:10.1016/j.conbuildmat.2010.09.042

[3] Y. El Hafiane, A. Smith, J. P. Bonnet, P. Abelard and P. Blanchart, "Electrical Characterization of Aluminous Cement at the Early Age in the $10 \mathrm{~Hz}-1 \mathrm{GHz}$ Frequency range," Cement and Concrete Research, Vol. 30, No. 7,
2000, pp. 1057-1062. doi:10.1016/S0008-8846(00)00285-4

[4] O. Nilufer, O. M. Thomas and P. S. Surendra, "NonDestructive Monitoring of Fiber Orientation Using AC-IS: An Industrial-Scale Application," Cement and Concrete Research, Vol. 36, No. 9, 2006, pp. 1653-1660. doi:10.1016/j.cemconres.2006.05.026

[5] Y. W. Leta, J. K. Neil, W. Supaporn and O. M. Thomas, "Combined Time Domain Reflectometry and AC-Impedance Spectroscopy of Fiber-Reinforced Fresh-Cement Composites," Cement and Concrete Research, Vol. 37, No. 1, 2007, pp. 89-95. doi:10.1016/j.cemconres.2006.09.004

[6] H. C. Schoenekess, W. Ricken, J.-G. Liu and W.-J. Becker, "Special Constructed and Optimised Eddy-Current Sensors for Measuring Force and Strain in Steel Reinforced Concrete," Sensors and Actuators A: Physical, Vol. 106, No. 1, 2003, pp. 159-163. doi:10.1016/S0924-4247(03)00156-0

[7] W Ahn and D. V. Reddy, "Galvanostatic Testing for the Durability of Marine Concrete under Fatigue Loading," Cement and Concrete Research, Vol. 31, No. 3, 2001, pp. 343-349. doi:10.1016/S0008-8846(00)00506-8

[8] Z. J. Li, X. S. Wei and W. L. Li, "Preliminary Interpretation of Hydration Process of Portland Cement Using Resistivity Measurement," ACI Materials Journal, Vol. 100, No. 3, 2003, pp. 253-257.

[9] N. R. Buenfeld, J. B. Newman and C. L. Page, "The Resistivity of Mortar Immersed in Sea-Water," Cement and Concrete Research, Vol. 16, No. 3, 1986, pp. 511-524. doi:10.1016/0008-8846(86)90089-X

[10] M. L. Shi, Z. Y. Chen and J. Sun, "Determination of Chloride Diffusivity in Concrete by AC Impedance Spectroscopy", Cement and Concrete Research, Vol. 29, No. 7, 1999, pp. 1111-1115. doi:10.1016/S0008-8846(99)00079-4

[11] S. Q. Li, J. S. Hu, B. Liu, G. H. Zhang, W. Cao, Q. Wang and N. Zhang, "Fundamental Study on Aluminophosphate Cement," Cement and Concrete Research, Vol. 29, No. 10,1999 , pp. 1549-1554. doi:10.1016/S0008-8846(99)00111-8 